\title{
A Non-Monotonic Goal Specification Language for Planning with Preferences
}

\author{
Tran Cao Son* and Enrico Pontelli* and Chitta Baral ${ }^{+1}$
}

\begin{abstract}
This paper introduces a default logic based approach to defining goal specification languages that can be non-monotonic and allow for the specification of inconsistencies and priorities among goals. The paper starts by presenting a basic goal specification language for planning with preferences. It then defines goal default theories (resp. with priorities) by embedding goal formulae into default logic (resp. prioritizing default logic). It is possible to show that the new language is general, as it can express several features of previously developed goal specification languages. The paper discusses how several other features can be subsumed by extending the basic goal specification language. Finally, we identify features that might be important in goal specification that cannot be expressed by our language.
\end{abstract}

\section{Introduction}

An important component of autonomous agent design is goal specification. In classical planning, goals deal with reaching one of a particular set of states. Nevertheless, often goals of agents are not just about reaching a particular state; goals are often about satisfying desirable conditions imposed on the trajectory. For example, a person can have the following desire in preparing travel plans to conferences:

(*) I prefer to fly to the conference site (since it is usually too far to drive).

The user's preference restricts the means that can be used in achieving her goal of reaching the conference site, which leads to the selection of a plan that reaches the conference site by airplane, whenever possible. Ultimately, this affects what actions the person should take in order to achieve the goal.

These observations led to the development of languages for the specification of soft goals in planning, e.g., $\mathcal{P} \mathcal{P}$ introduced in [14] and modified in [6]. In $\mathcal{P} \mathcal{P}$, a basic desire is a temporal formula describing desirable properties of a plan. Atomic and general preferences are particular classes of formulae built over basic desires. A preference formula $\Phi$ defines a preference order $\prec_{\Phi}$ among the trajectories that achieve the hard goal of the problem, i.e., for every pair of trajectories $\alpha$ and $\beta, \alpha \prec_{\Phi} \beta$ indicates that $\alpha$ is preferable to $\beta$. $\prec_{\Phi}$ is often a partial order and its definition relies on the notion of satisfaction between trajectories and a preference specification. Similar ideas have been considered in the planning community and led to extensions of the planning domain description language PDDL, with features for representing classes of preferences over plans using temporal extended preferences (e.g., [10]).

\footnotetext{
$1 *$ Department of Computer Science New Mexico State University, Las Cruces, New Mexico, USA, email: tson epontell@cs.nmsu.edu and ${ }^{+}$Department of Computer Science and Engineering, Arizona State University, Tempe, Arizona, USA, email: chitta@asu .edu
}

In [4], the authors argue that a goal specification language should be non-monotonic for various reasons, such as elaboration tolerance and simplicity of goal specification. For example, the same traveler with the preference $(*)$ would probably not mind driving at most three hours to the conference site if the only flight to the destination requires to travel the day before the conference starts. In this case, her preference becomes:

(**) Normally, I prefer to fly to the conference site (since it is usually too far to drive). However, if there are no flights on the same day of the conference and the driving time is at most three hours, then I will drive.

To address this issue, an extension of LTL [11], called N-LTL, has been proposed, allowing weak and strong exceptions to certain rules. A weakness of this language is that it requires the classification of weak and strong exceptions when a goal is specified. In [5], the language ER-LTL is introduced to address this limitation of N-LTL. Similarly to $\mathcal{P} \mathcal{P}$, the semantics of N-LTL and ER-LTL relies on the notion of satisfaction between plans and N-LTL or ER-LTL specifications. Observe that the issue of non-monotonicity is dealt within $\mathcal{P} \mathcal{P}$ and in the extensions of PDDL by revising the soft goals, which is an approach that N-LTL specifically tries to avoid.

We observe that the focus of the work in $[1,4,5,6,10]$ is on classical planning, i.e., the planning domains are deterministic and the initial state is complete, while the work in [14] considers nondeterministic domains and only discusses preferences among weak plans. In [2], it is argued that plans for non-deterministic domains should be policies (i.e., a partial function from the set of states to the set of actions) and the language $\pi$-CTL ${ }^{*}$ is developed for specifying goals in non-deterministic domains. $\pi$-CTL ${ }^{*}$ is an extension of CTL ${ }^{*}$ [9] with two modalities $A_{\pi}$ and $E_{\pi}$ for considering all or some trajectories w.r.t. a given policy. In [3], the language $\pi$-CTL ${ }^{*}$ is extended with quantifiers over policies to increase its expressiveness. Policies satisfying a goal specification are viewed as the solutions of a planning problem.

In this paper, we explore an approach based on prioritizing default logic for defining a goal specification language. The new language, called goal default theories with priorities, is a variation of prioritizing default logic, in which formulae occurring within a default can be temporal extended preference formulae. We show that the core of the new language subsumes several features from existing goal languages and can be extended to subsume several other features from other goal languages. Finally, we discuss the possible applications of the new language in the study of existing goal languages and the development of new ones.

\section{Background}

In this section, we briefly review the basic definitions of planning, linear temporal logic (LTL) and its extension for specifying prefer- 
ences in planning.

\subsection{LTL and Temporal Extended Preferences}

Let $\mathcal{L}$ be a propositional language. By $\langle p\rangle$ we denote a propositional formula from $\mathcal{L}$. LTL-formulae are defined by the following syntax

$$
\begin{aligned}
\langle f\rangle::= & \langle p\rangle|\langle f\rangle \wedge\langle f\rangle|\langle f\rangle \vee\langle f\rangle \mid \\
& \neg\langle f\rangle|\bigcirc\langle f\rangle| \square\langle f\rangle|\diamond\langle f\rangle|\langle f\rangle \mathrm{U}\langle f\rangle
\end{aligned}
$$

The semantics of LTL-formulae is defined with respect to sequences of interpretations of $\mathcal{L}$. For later use, we will refer to an interpretation of $\mathcal{L}$ as a state and a possibly infinite sequence of interpretations of $\mathcal{L}, s_{0}, s_{1}, \ldots$, as a trajectory. For a trajectory $\sigma=s_{0}, s_{1}, \ldots$, by $\sigma_{i}$ we denote the suffix $s_{i}, s_{i+1}, \ldots$ of $\sigma$. A trajectory $\sigma=s_{0}, s_{1}, \ldots$ satisfies an LTL-formula $f$, denoted by $\sigma \mid=f$, if $\sigma_{0} \mid=f$ where

- $\sigma_{j} \models p$ iff $s_{j} \models p$

- $\sigma_{j}=\neg f$ iff $\sigma_{j} \not \models f$

- $\sigma_{j}=f_{1} \wedge f_{2}$ iff $\sigma_{j} \models f_{1}$ and $\sigma_{j} \models f_{2}$

- $\sigma_{j}=f_{1} \vee f_{2}$ iff $\sigma_{j} \models f_{1}$ or $\sigma_{j} \models f_{2}$

- $\sigma_{j}=\bigcirc f$ iff $\sigma_{j+1}=f$

- $\sigma_{j}=\square f$ iff $\sigma_{k}=f$, for all $k \geq j$

- $\sigma_{j}=\diamond f$ iff $\sigma_{i} \models f$ for some $i \geq j$

- $\sigma_{j} \models f_{1} \cup f_{2}$ iff there exists $k \geq j$ such that

$\sigma_{k}=f_{2}$ and for all $i, j \leq i<k, \sigma_{i}=f_{1}$.

A finite trajectory $s_{0}, \ldots, s_{n}$ satisfies an LTL-formula $f$ if its extension $s_{0}, \ldots, s_{n}, s_{n+1}, \ldots$ satisfies $f$, where $s_{k}=s_{n}$ for $k>n$. In order to deal with planning problems, LTL is extended with the following constructs

$$
\begin{gathered}
\text { at_end }\langle p\rangle \mid\langle p\rangle \text { sometime_before }\langle p\rangle \mid \\
\langle p\rangle \text { sometime_after }\langle p\rangle
\end{gathered}
$$

Formulae of the extended LTL are referred to as Temporal Extended Preferences (TEP). Note that the last two are syntactic sugars for LTL formulae. Temporal extended preferences are interpreted over finite trajectories. The notion of satisfaction for standard LTL-formulae is defined as above, while satisfaction of TEP formulae is as follows: given a finite trajectory $\sigma=s_{0}, \ldots, s_{n}$ :

- $\sigma \models$ at_end $p$ iff $s_{n} \models p$;

- $\sigma \models p_{1}$ sometime_before $p_{2}$ iff for every $i, 0 \leq i \leq n$, if $\sigma_{i} \mid=p_{1}$ then $\sigma_{j}=p_{2}$ for some $i \leq j \leq n$; and

- $\sigma_{j} \models p_{1}$ sometime_after $p_{2}$ iff for every $i, 0 \leq i \leq n$, if $\sigma_{i} \mid=p_{1}$ then $\sigma_{j} \mid=p_{2}$ for some $0 \leq j<i \leq n$.

\subsection{Planning}

In this paper, we describe a dynamic domain as a labeled transition system $T=(F, A, S, L)$, where:

- $F$ is a set of fluents (or propositions),

- $A$ is a set of actions,

- $S$ is a set of interpretations (or states) of $F$, and

- $L \subseteq S \times A \times S$.

Each triple $\left\langle s_{1}, a, s_{2}\right\rangle \in L$ indicates that the execution of the action $a$ in the state $s_{1}$ might result in the state $s_{2} . T$ is deterministic if for each state $s$ and action $a, L$ contains at most one triple $\left\langle s, a, s_{2}\right\rangle$; otherwise, $T$ is non-deterministic.

Given a transition system $T$, a finite or infinite sequence $s_{0} a_{0} s_{1} a_{1} \ldots s_{n} a_{n} s_{n+1} \ldots$ of alternate states and actions is called a run if $\left\langle s_{i}, a_{i}, s_{i+1}\right\rangle \in L$ for every $i=0, \ldots$ A policy $\pi$ in a transition system $T$ is a partial function $\pi: S \rightarrow A$ from the set of states to the set of actions. A run $s_{0} a_{0} s_{1} a_{1} \ldots s_{k} a_{k} s_{k+1} \ldots$ is said to be induced by a policy $\pi$ if $a_{i}=\pi\left(s_{i}\right)$ for every $i=0, \ldots, k, \ldots$

Definition 1. A planning problem is a triple $\left\langle T, S_{i}, S_{f}\right\rangle$ where $T=$ ( $F, A, S, L)$ is a transition system, $S_{i} \subseteq S$ is the set of initial states, and $S_{f} \subseteq S$ is the set of final states.

Intuitively, a planning problem asks for a plan which transforms the transition system from any state belonging to $S_{i}$ to some state in $S_{f}$. In the rest of the discussion, we assume $S_{i}$ and $S_{f}$ to be finite sets. We distinguish two classes of planning problems:

- Deterministic planning: in this case, $T$ is deterministic and a solution (or plan) of $\left\langle T, S_{i}, S_{f}\right\rangle$ is an action sequence $\left[a_{0} ; \ldots ; a_{n}\right]$ such that, for every $s_{0} \in S_{i}, s_{0} a_{0} s_{1} a_{1} \ldots a_{n} s_{n+1}$ is a run in $T$ and $s_{n+1} \in S_{f}$;

- Non-deterministic planning: in this case, $T$ is non-deterministic and a solution (or plan) of $\left\langle T, S_{i}, S_{f}\right\rangle$ is a policy $\pi$ such that, for every $s_{0} \in S_{i}$ and every run induced by $\pi$ in $T, \pi$ is finite and is of the form $s_{0} a_{0} s_{1} a_{1} \ldots s_{k} a_{k} s_{k+1}$ where $s_{k+1} \in S_{f}$.

In the following, whenever we refer to a possible plan in a transition system $T$, we mean a sequence of actions (resp. a policy) if $T$ is deterministic (resp. non-deterministic) that can generate a correct run. Let us illustrate these basic definitions using the following simple example.

Example 1. Consider a transportation robot. There are different locations, say $l_{1}, \ldots, l_{k}$, whose connectivity is given by a graph and there might be different objects at each location. Let $O$ be a set of objects. The robot can travel between two directly connected locations. It can pick up objects at a location, hold them, drop them, and carry them between locations. We assume that, for each pair of connected locations $l_{i}$ and $l_{j}$, the robot has an action $a_{i, j}$ for traveling from $l_{i}$ to $l_{j}$. The robot can hold only one object at a time. The domain can be represented by a transition system $T_{1}=(F, A, S, L):^{2}$

- F contains the following types of propositions:

- at $(i)$ denotes that the robot is at the location $l_{i}$;

- o_at $(o, i)$ denotes that the object o is at the location $l_{i}$;

- $h(o)$ denotes that the robot is holding the object o.

- A contains of the following types of actions:

- $a_{i, j}$ the robot moves from $l_{i}$ to $l_{j}$;

- release (o) the robot drops the object o;

- pickup $(o)$ the robot picks up the object $o$.

- $S$ contains the interpretations of $F$ which satisfy the basic constraints, such as the robot is at one location at a time, it holds only one object, etc.

- $L$ contains transitions of the form $\left\langle s, a, s^{\prime}\right\rangle$ such that $s^{\prime}$ is the result of the execution of $a$ in $s$; for example, if $a=a_{i, j}$ and at $(i) \in$ s then $s^{\prime}=s \backslash\{a t(i)\} \cup\{a t(j)\}$.

$T_{1}$ is a deterministic transition system. We will also refer to $T_{2}$ as the non-deterministic version of $T_{1}$ by defining $T_{2}=\left(F, A, S, L^{\prime}\right)$ where $L^{\prime}=L \cup\left\{\left\langle s_{i}, a_{i, j}, s_{i}\right\rangle \mid a_{i, j} \in A\right\}$ and at $(i) \in$ s. Intuitively, $T_{2}$ encodes the fact that the action $a_{i, j}$ might fail and, when it does, the robot will stay where it was after the execution of $a_{i, j}$.

A planning problem $P$ in this domain is given by specifying the initial location of the robot and of the objects and the final location of the robot and of the objects. It is deterministic (resp. nondeterministic) if $T_{1}$ (resp. $T_{2}$ ) is considered.

For example, $P_{i}=\left\langle T_{i},\{\{a t(1)\}\}, S_{f}\right\rangle$ where for each $s \in S_{f}$, at $(k) \in s$ is a planning problem for $T_{i}$. A solution for $P_{1}$ is a sequence $\left[a_{1,2} ; \ldots ; a_{k-1, k}\right]$. On the other hand, a solution for $P_{2}$ is a policy $\pi$ defined by $\pi(s)=a_{t, t+1}$ iff at $(t) \in$ sor $t<k$.

${ }^{2}$ We simplify the definitions of $S$ and $L$ for readability. 


\section{A Basic Goal Specification Language for Planning with Preferences}

In the literature, a planning problem with preferences is defined as a pair $(P, \Phi)$ of a planning problem $P=\left\langle T, S_{i}, S_{f}\right\rangle$, where $T=(F, A, S, L)$, and a preference formula $\Phi$ in a goal specification language. A plan $\delta$ of $P$ is called a preferred plan if it is a plan for $P$ and satisfies $\Phi$, where the notion of satisfaction of a preference formula by a plan is language dependent.

In general, we can characterize a goal specification language $\mathcal{G}$ over a transition system $T$ by a set of preference formulae $\mathcal{F}$ and a satisfaction relation $\models_{\mathcal{G}}$ between the set of possible plans of $T$ and formulae in $\mathcal{F}$. We will write $\delta=_{\mathcal{G}} \Phi$ to denote that the plan $\delta$ satisfies the formula $\Phi$ under the language $\mathcal{G}$.

For later use, we will define a basic goal specification language for a transition system $T=(F, A, S, L)$, written as $\mathcal{G}_{b}=\left(\mathcal{F}_{b}, \mid=\mathcal{G}_{b}\right)$, as follows:

- the set of preference formulae $\mathcal{F}_{b}$ is the set of TEP-formulae over $F \cup A$, and

- for a planning problem $P=\left\langle T, S_{i}, S_{f}\right\rangle, \models_{\mathcal{G}_{b}}$ is defined as follows:

$\circ$ if $T$ is deterministic, a plan $\delta=\left[a_{0}, \ldots, a_{n}\right]$ for a planning problem $P$ is said to satisfy a formula $\Phi$ in $\mathcal{F}_{b}$ if for every $s_{0} \in S_{i}, s_{0} a_{0} s_{1} a_{1} \ldots a_{n} s_{n+1}$ is a run in $T$ and $\left(s_{0} \cup\right.$ $\left.\left\{a_{0}\right\}\right), \ldots,\left(s_{n} \cup\left\{a_{n}\right\}\right), s_{n+1}$ is a trajectory satisfying $\Phi$ (in the TEP-language over $F \cup A$ );

$\circ$ if $T$ is non-deterministic, a solution (policy) $\pi$ for $P$ is said to satisfy a formula $\Phi$ in $\mathcal{F}_{b}$ if for every $s_{0} \in S_{i}$ and every run $s_{0} a_{0} s_{1} a_{1} \ldots s_{k} a_{k} s_{k+1}$ in $T$ induced by $\pi,\left(s_{0} \cup\right.$ $\left.\left\{a_{0}\right\}\right), \ldots,\left(s_{n} \cup\left\{a_{n}\right\}\right), s_{n+1}$ is a trajectory satisfying $\Phi$ (in the TEP-language over $F \cup A$ ).

In the following, we will assume that any goal specification language $\mathcal{G}$ is a conservative extension of $\mathcal{G}_{b}$, i.e., (i) $\mathcal{G}$ contains all formulae in $\mathcal{G}_{b}$; and (ii) for every planning problem $P$ and a formula $\Phi$ in $\mathcal{G}$, if $\Phi \in \mathcal{G}_{b}$ and $\delta \models \models_{\mathcal{G}_{b}} \Phi$ with respect to $\mathcal{G}_{b}$ then $\delta \models_{\mathcal{G}} \Phi$ with respect to $\mathcal{G}$.

Example 2. Some preference formulae in $\mathcal{G}_{b}$ for the transition systems in Ex. 1 are:

- $\diamond$ at (2): the robot should visit the location $l_{2}$ during the execution of the plan;

- at $(1) \wedge \diamond a t(2):$ the robot must (i) start in a state satisfying at (1) (or the robot is at the location $l_{1}$ initially); and (ii) visit the location $l_{2}$ at some point during the execution of the plan;

- $\square\left[a t(2) \Rightarrow\left(\bigvee_{i \neq 2} a_{2 i}\right)\right]:$ whenever the robot visits $l_{2}$, it should leave that location immediately by executing an action going to one of its neighbors;

- $h(o) \Rightarrow \bigcirc \bigcirc \neg h(o)$ : if the robot holds an object o in the initial state then it should release o after the execution of one action;

- $\square[h(o) \Rightarrow \bigcirc \bigcirc \neg h(o)]$ : whenever the robot holds an object o it should release o after the execution of an action;

- $h(o)$ sometime_before at(5): whenever the robot holds the object $o$, it must visit the location $l_{5}$ thereafter before reaching the goal;

- at_end $\left[\bigwedge_{o \in O} \neg h(o)\right]$ : at the end, the robot should not hold any object.

With a slight abuse of notation, let us view a state $s$ as a formula $\bigwedge_{s \models f} f \wedge \bigwedge_{s \models \neg f} \neg f$. Let $S_{i}$ and $S_{f}$ be two sets of states and

$$
\Phi=[\underbrace{\bigvee_{s \in S_{i}} s \wedge \text { at_end }\left[\bigvee_{s \in S_{f}} s\right]}_{\Phi_{1}} \underbrace{\bigvee_{S_{2}}}_{\Phi_{2}}]
$$

It is easy to see that any plan satisfying $\Phi$ requires its execution to start from a state satisfying $\Phi_{1}$, which is one of the states in $S_{i}$, and end in a state satisfying $\Phi_{2}$, which is one of the states in $S_{f}$. For this reason, the description of the initial and final states can be folded into a preference formula. We will therefore define planning problems as follows.

Definition 2. Given a transition system $T$ and a goal specification language $\mathcal{G}=\left(\mathcal{F}, \models_{\mathcal{G}}\right)$ over $T$, a goal formula $\Phi$ in $\mathcal{F}$ is called a planning problem. A solution of $\Phi$ is a plan $\delta$ in $T$ such that $\delta \models_{\mathcal{G}} \Phi$.

By Def. 2, a goal formula represents a planning problem. The literature is quite diversified when a user faces two or more goal formulae which are contradictory with each other. For example, the formula $\diamond a t(2)$ is contradictory with $\square \neg a t(2)$; $\square \neg\left(\bigwedge_{o \in O} h(o)\right)$ conflicts with $\diamond h\left(o_{1}\right)$; etc. A possibility is to consider a possible plan as solution if it satisfies some goal formulae. Another possibility is to rank the goal formulae and identify solutions as plans that satisfy the formula with the highest possible ranking. In the following, we will show that a uniform framework for dealing with conflicting goal formulae can be obtained by embedding goal formulae into Reiter's default logic.

\section{Goal Default Theories}

In this section, we will introduce a new goal specification language, called goal default theory. A goal default theory is a variation of Reiter's default theory [12], whose defaults can contain preference formulae. Goal default theories provide a possible treatment of planning with multiple goal formulae.

A goal default theory is defined over a transition system $T=$ $(F, A, S, L)$ and a goal specification language $\mathcal{G}=\left(\mathcal{F},\left.\right|_{\mathcal{G}}\right)$.

Given a goal specification language $\left(\mathcal{F}, \models_{\mathcal{G}}\right)$, we say that two formulae $\varphi, \psi$ in $\mathcal{F}$ are equivalent w.r.t. $=_{\mathcal{G}}$ if, for each plan $\delta$ of $T$, we have that $\delta \models_{\mathcal{G}} \varphi \Leftrightarrow \psi{ }^{3}$ We can easily extend this notion to

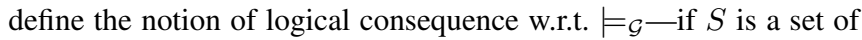
formulae from $\mathcal{F}$ and $f$ is another formula in $\mathcal{F}$, then $S \models_{\mathcal{G}} f$ if for each plan $\delta$ of $T$ we have that $\delta \models_{\mathcal{G}} \bigwedge_{\varphi \in S} \varphi$ implies $\delta \models_{\mathcal{G}} f$. Given a set of formulae $S$, we define $\operatorname{Decl}(S)=\left\{\varphi \mid \varphi \in \mathcal{F}, S \models_{\mathcal{G}} \varphi\right\}$.

A preference default (or $p$-default) $d$ over $\mathcal{G}$ is of the following form

$$
\frac{\alpha: \beta}{\gamma}
$$

where $\alpha, \beta$, and $\gamma$ are formulae in $\mathcal{F}$. We call $\alpha$ the precondition, $\beta$ the justification, and $\gamma$ the consequence of $d$, and we denote them with $\operatorname{prec}(d), j u s t(d)$, and cons $(d)$, respectively. A default $d$ is said to be

- Normal if its justification is equivalent to its conclusion;

- Prerequisite-free if its precondition is equivalent to true; and

- Supernormal if it is normal and prerequisite-free.

Given a set of formulae $S$ from $\mathcal{F}$, a default $d$ is said to be defeated in $S$ if $S=\neg j u s t(d)$. Some preferences and their representation as p-defaults over $\mathcal{G}_{b}$ for the domain from Example 1 are given next.

Example 3. In these examples, o denotes a particular object in the domain. 
- If there is no evidence that the robot is initially at the location $l_{2}$, then it should go to $l_{2}$ :

$$
\frac{\top: \neg a t(2)}{\diamond a t(2)}
$$

- Assume that objects might be defective, represented by the proposition defective. We can write

$$
\frac{\top: \square[\neg \text { defective }(o)]}{\square[a t(2) \Rightarrow h(o)]}
$$

to indicate that normally, we would like that the robot holds the object o whenever it is at the location $l_{2}$. An exception to this rule is possible if the object o is defective.

- If the robot is not required to hold the object o in the final state and there is no evidence that it initially holds o, then it should not execute the action of picking up the object o:

$$
\frac{\top \quad: \quad \text { at_end }(\neg h(o)) \wedge \neg h(o)}{\square[\neg \operatorname{pickup}(o)]}
$$

- If there is no evidence that the object o is initially in the wrong place then the robot should not start by executing the action of picking up the object $\mathrm{o}$ :

$$
\frac{\text { at_end }\left(o_{-} a t(o, i)\right): \bigwedge_{i \neq j} \neg o_{-} a t(o, j)}{\neg \operatorname{pickup}(o)}
$$

- A stronger version of (7) is

$$
\frac{\text { at_end }\left(o \_a t(o, i)\right): \bigwedge_{i \neq j} \neg o_{-} a t(o, j)}{\square \neg p i c k u p(o)}
$$

indicates that the robot should never pick up the object o if o could already be in the desired final location.

- If there is the possibility that the robot might reach location $l_{2}$, then it must leave the location immediately after its arrival at $l_{2}$.

$$
\frac{T: \diamond[a t(2)]}{\square\left[a t(2) \Rightarrow \bigcirc \bigvee_{i \neq 2} a_{2, i}\right]}
$$

- If there is no evidence that an object o will ever appear in location $i$ then the robot should never go there.

$$
\frac{\top: \square\left[\neg o_{-} a t(o, i)\right]}{\square\left[\bigvee_{j \neq i} \neg a_{j, i}\right]}
$$

In the following, we will refer to the p-defaults in (4)-(9) by $p_{1}, \ldots, p_{6}$, respectively.

We next define the notion of a goal default theory.

Definition 3. A goal default theory over a goal language $\mathcal{G}=$ $\left(\mathcal{F}, \mid=_{\mathcal{G}}\right)$ and a transition system $T$ is a pair $\Sigma=(D, W)$ where $D$ is a set of p-defaults over $\mathcal{G}$ and $W \subseteq \mathcal{F}$.

Given a set of p-defaults $D$, we denote with $\operatorname{cons}(D)$ the set $\operatorname{cons}(D)=\{\operatorname{cons}(d) \mid d \in D\}$. A p-default $d$ is applicable w.r.t. a set of $\mathcal{F}$ formulae $S$ if $S \models_{\mathcal{G}} \operatorname{prec}(d)$ and $S \not \models_{\mathcal{G}} \neg j u s t(d)$. Let us denote with $\Pi_{D}(S)$ the set of p-defaults from $D$ that are applicable w.r.t. $S$.

Definition 4 (From [12]). Let $\Sigma=(D, W)$ be a goal default theory over $\mathcal{G}=\left(\mathcal{F}, \mid=_{\mathcal{G}}\right)$ and $T$. An extension of $\Sigma$ is a minimal set $E \subseteq \mathcal{F}$ that satisfies the condition $E=\operatorname{Decl}\left(W \cup \operatorname{Cons}\left(\Pi_{D}(E)\right)\right)$. We say that $\Sigma$ is consistent if it has at least one extension.

From this definition, any default over the propositional language
$F \cup A$ is a p-default, and any Reiter's default theory over the language $F \cup A$ is a goal default theory.

Definition 5. Given a transition system $T=(F, A, S, L)$ and a goal specification language $\mathcal{G}=\left(\mathcal{F}, \models_{\mathcal{G}}\right)$ over $T$, a planning problem over $T$ and $\mathcal{G}$ is a goal default theory $\Sigma=(D, W)$ over $\mathcal{G}$ and $T$.

The notion of a solution to a planning problem is modified as follows.

Definition 6. Given a transition system $T=(F, A, S, L)$, a goal specification language $\mathcal{G}=\left(\mathcal{F}, \models_{\mathcal{G}}\right)$ over $T$, and a planning problem $\Sigma$ over $T$ and $\mathcal{G}, a$ solution of $\Sigma$ is a plan $\delta$ in $T$ such that $\delta \models_{\mathcal{G}}$ E for some extension $E$ of $\Sigma$.

Some planning problems over the transition systems in Exp. 1 and the language $\mathcal{G}_{b}$ are given in the next example.

Example 4 (Continuation of Example 3). - Let $\Sigma_{1}=$ $\left(\left\{p_{1}\right\},\{a t(1)\right.$, at_end at $\left.(5)\}\right)$ where $p_{1}$ is the default (4). Intuitively, we have that $\Sigma_{1}$ identifies plans where the robot starts at location $l_{1}$, goes through the location $l_{2}$, and ends in location $l_{5}$.

- Let $\Sigma_{2}=\left(\left\{p_{6}\right\},\{a t(1)\right.$, at_end at $\left.(5)\}\right)$ where $p_{6}$ is the default (9). This identifies plans where the robot starts at location $l_{1}$, ends in location $l_{5}$, and either (i) never goes through the location $l_{2}$; or (ii) never stays in the location $l_{2}$ within two consecutive steps. $\square$

The planning problems in Example 4 are simple, in that they are specified by goal default theories whose set of defaults is a singleton. Let us consider a more complicated example. Assume that we have two temporal formulae $\Phi$ and $\Psi$ such that there exists no plan that can satisfy both $\Phi$ and $\Psi$. In this case, the use of goal default theory as a goal formula comes in handy. Indeed, every solution of the planning problem expressed by the goal default theory

$$
\Sigma_{\Phi, \Psi}=\left(\left\{\frac{\top: \neg \Psi}{\Phi}, \frac{\top: \neg \Phi}{\Psi}\right\}, \emptyset\right)
$$

satisfies either $\Phi$ or $\Psi$. The following result generalizes this observation.

Proposition 1. Let $T=(F, A, S, L)$ be a transition system, $\mathcal{G}=$ $\left(\mathcal{F}, \models_{\mathcal{G}}\right)$ be a goal specification language, and $\Delta=\left\{\Phi_{1}, \ldots, \Phi_{n}\right\}$ be a set of preference formulae in $\mathcal{F}$. Furthermore, let

$$
\Sigma_{\Delta}=\left(\left\{\frac{T: \Psi}{\Psi} \quad \mid \quad \Psi \in \Delta\right\}, \emptyset\right)
$$

- For every solution $\delta$ to the problem $\Sigma_{\Delta}$ there exists a maximal (w.r.t. $\subseteq$ ) set of preferences $\Delta_{\delta} \subseteq \Delta$ such that $\delta \models_{\mathcal{G}} \bigwedge_{\Psi \in \Delta_{\delta}} \Psi$;

- For every pair of solutions $\delta$ and $\delta^{\prime}$ of $\Sigma_{\Delta}$, either $\Delta_{\delta}=\Delta_{\delta^{\prime}}$ or $\Delta_{\delta} \not \subset \Delta_{\delta^{\prime}}$ and $\Delta_{\delta^{\prime}} \not \subset \Delta_{\delta}$.

\section{Goals Default Theories with Priorities}

Proposition 1 shows that goal default theories can be used to specify planning problems with multiple preferences which might not be consistent with each other. For instance, consider a traveler from New York to San Francisco who has two preferences: reach the destination as fast as possible $\left(\Phi_{1}\right)$ and spend the least amount of money $\left(\Phi_{2}\right)$. In general, these two preferences cannot be satisfied at the same time. In this case, it is more reasonable to assume that a plan satisfying one of the criteria is an acceptable solution. Thus, $\Sigma_{\left\{\Phi_{1}, \Phi_{2}\right\}}$ is a reasonable goal specification if the traveler is impartial about $\Phi_{1}$ and $\Phi_{2}$. On the other hand, if the traveler prefers $\Phi_{1}$ over $\Phi_{2}$ (or vice versa), we will need to change the goal specification or provide additional ways for the traveler to specify this priority. As it turns out, the literature is rich with approaches for adding priorities to default theories [7, 8] which can be easily adapted to goal default theories. We next define 
goal default theories with priorities by adapting the work of [7] to goal default theories.

Let us start by introducing static priorities, encoded by a wellordering relation $\prec$ among $\mathrm{p}$-defaults-i.e., $\prec$ is transitive, irreflexive, and each set of elements admits the least element in the ordering. We denote with $\min _{\prec}(X)$ the least element of $X$ with respect to $\prec$. We define goal default theory with priorities as follows.

Definition 7. A goal default theory with priorities over a goal language $\mathcal{G}=\left(\mathcal{F}, \models_{\mathcal{G}}\right)$ and a transition system $T$ is a triple $(D, W, \prec)$ where $D$ is a set of p-defaults over $\mathcal{G}, \prec$ is a well-ordering relation over $D$, and $W \subseteq \mathcal{F}$.

Following the general design of prioritizing default theory [7], the notion of preferred extension can be defined by successively simplifying the structure of the defaults.

Let us identify a construction of preferred extension through the application of defaults according to the ordering imposed by $\prec$. Let us introduce the $\mathcal{P} \mathcal{R}$ operator which computes the next "preferred" set of goal formulae from an existing one:

- $\mathcal{P} \mathcal{R}_{\prec}(S)=\operatorname{Decl}(S \cup\{\operatorname{cons}(d)\})$

$$
\text { if } \Pi_{D}^{*}(S) \neq \emptyset \wedge d=\min _{\prec}\left(\left\{x \mid x \in \Pi_{D}^{*}(S)\right\}\right) ;
$$

- $\mathcal{P} \mathcal{R}_{\prec}(S)=S$ if $\Pi_{D}^{*}(S)=\emptyset$

where $\Pi_{D}^{*}(S)=\left\{d \mid d \in \Pi_{D}(S), S \not \forall \operatorname{cons}(d)\right\}$. If the elements in $D$ (for a goal default theory $(D, W)$ ) are supernormal, then it is possible to use $\mathcal{P} \mathcal{R}_{\prec}$ to produce a monotone sequence of goal formulae, by setting $S_{0}=\operatorname{Decl}(W), S_{i+1}=\mathcal{P} \mathcal{R}_{\prec}\left(S_{i}\right)$ for any successor ordinal $i+1$ and $S_{i}=\operatorname{Decl}\left(\bigcup_{j<i} S_{j}\right)$ for any limit ordinal $i$. We will denote the result of this construction as Pref $f_{\prec}(D, W)=\bigcup_{i \geq 0} S_{i}$.

The process of determining a preferred extension will apply Pref $f_{\prec}$ on a reduced version of the theory, in a style similar to that used in the Gelfond-Lifschitz reduct. Following the model proposed in [7], the reduct of a goal default theory with priorities $(D, W, \prec)$ w.r.t. a set of goal formulae $S$, denoted $\left(D^{S}, W, \prec^{S}\right)$, is obtained as follows:

- Determine $D^{\prime}=\left\{\frac{T: j u s t(d)}{\operatorname{cons}(d)} \mid d \in D, S \models_{\mathcal{G}} \operatorname{prec}(d)\right\}$

- Determine $D^{S}=\left\{d \in D^{\prime} \mid \operatorname{cons}(d) \notin S\right.$ or $\left.S \not \forall_{\mathcal{G}} \neg j u s t(d)\right\}$ and $\prec^{S}$ is such that $d_{1}^{\prime} \prec^{S} d_{2}^{\prime}$ if $d_{1} \prec d_{2}$ and $d_{1}\left(d_{2}\right)$ is the $\prec$-least element that introduced $d_{1}^{\prime}\left(d_{2}^{\prime}\right)$ in $D^{\prime}$.

We define preferred extensions as follows.

Definition 8. Let $(D, W, \prec)$ be a goal default theory with priorities over $\mathcal{G}=\left(\mathcal{F}, \models_{\mathcal{G}}\right)$ and $T$. A preferred extension $E$ of $(D, W, \prec)$ is a set of goal formulae in $\mathcal{F}$ such that $E$ is an extension of $(D, W)$ and $E=\operatorname{Pref}_{\prec E}\left(D^{E}, W\right)$.

Similar to [7], we can generalize the above definitions and define (i) a goal default theory with priorities as a triple $(D, W, \prec)$ where $(D, W)$ is a goal default theory and $\prec$ is a partial order among defaults in $D$; and (ii) a set of formulae $E$ is a preferred extension of $(D, W, \prec)$ if it is a preferred extension of some $\left(D, W, \prec_{E}\right)$ for some well-ordering $\prec_{E}$ which is an extension of $\prec$. For brevity, we omit the precise definitions. Definitions 5 and 6 can be extended in the obvious way: a planning problem is a goal default theory with priorities $(D, W, \prec)$ and its solutions are preferred extensions of $(D, W, \prec)$.

Example 5. Let us consider the domain in Example 1. Let us assume that, among the objects, there is a very valuable object $o_{1}$ and a dangerous object $\mathrm{O}_{2}$. Furthermore, let us assume that the robot is equipped with actions that can detect the object $\mathrm{O}_{2}$ whenever the robot is at the same location as $\mathrm{O}_{2}$. However, the equipment might not be working. We will denote with working the fact that the equipment is working properly. Let us consider the two formulae:
- $\varphi:=\diamond h\left(o_{1}\right):$ the robot should try to get the object $o_{1}$

- $\psi:=\square\left[\bigwedge_{i \in\{1, \ldots, k\}}\left(o_{-} a t\left(o_{2}, i\right) \Rightarrow \neg a t(i)\right)\right]$ : the robot should not be at the same place with object $\mathrm{O}_{2}$ at any time.

With these formulae, we can define the following p-defaults:

$$
g_{1} \equiv \frac{\top: \text { working }}{\psi \wedge \varphi} \quad g_{2} \equiv \frac{\top: \neg \text { working }}{\varphi}
$$

$g_{1}$ indicates that if the equipment is initially working, then the robot will get $o_{1}$ while trying to avoid $o_{2} . g_{2}$ states that if the equipment is not working, then the robot will only worry about getting $o_{1}$. The theory $\left(\left\{g_{1}, g_{2}\right\}, \emptyset,\left\{g_{1} \prec g_{2}\right\}\right)$ states that we prefer that the robot tries to satisfy $g_{1}$ before trying to satisfy $g_{2}$.

\section{Related Work and Discussion}

In this section, we relate goal default theories with priorities to existing goal specification languages. We then discuss possible applications of the new language.

- TEP formulae: TEP formulae have been implemented in a planner in [1]. Given a set of TEP formulae $\Delta=\left\{\Phi_{1}, \ldots, \Phi_{n}\right\}$, a planning problem is an optimization problem that maximizes the rewards obtained by satisfying the formulae in $\Delta$. Formally, the reward over a plan $\delta$ is

$$
\Sigma_{\Phi_{i} \in \Delta, \delta \models \Phi_{i}} \operatorname{reward}\left(\Phi_{i}\right)-\Sigma_{\Phi_{i} \in \Delta, \delta \mid \not \Phi_{i}} \text { penalty }\left(\Phi_{i}\right)
$$

where $\operatorname{reward}(\Phi)$ and penalty $(\Phi)$ denote the reward and penalty for satisfying and not satisfying $\Phi$, respectively.

The planning problem can be expressed by a goal default theory with priorities as follows. Let $S$ be a set of formulae, $S \subseteq \Delta$, and $d_{S}$ be the default

$$
\frac{\top: \bigwedge_{\Phi \in S} \Phi \wedge \bigwedge_{\Phi \in \Delta \backslash S} \neg \Phi}{\bigwedge_{\Phi \in S} \Phi \wedge \bigwedge_{\Phi \in \Delta \backslash S} \neg \Phi}
$$

Let $D_{\Delta}=\left\{d_{S} \mid S \subseteq \Delta\right\}$ and $\prec \Delta$ be the partial order over $D_{\Delta}$ where $d_{S} \prec \Delta d_{S^{\prime}}$ if

$$
\begin{aligned}
& \Sigma_{\Phi_{i} \in S} \operatorname{reward}\left(\Phi_{i}\right)-\Sigma_{\Phi_{i} \notin S \text { penalty }}\left(\Phi_{i}\right) \geq \\
& \Sigma_{\Phi_{i} \in S^{\prime}} \operatorname{reward}\left(\Phi_{i}\right)-\Sigma_{\Phi_{i} \notin S^{\prime}} \text { penalty }\left(\Phi_{i}\right) .
\end{aligned}
$$

We can show that $\left(D_{\Delta}, \emptyset, \prec_{\Delta}\right)$ is a goal default theory with priorities representing the given planning problem, i.e., any preferred solution of $\left(D_{\Delta}, \emptyset, \prec_{\Delta}\right)$ is a solution of the original planning problem and vice versa.

- $\mathcal{P} \mathcal{P}$ : The language $\mathcal{P} \mathcal{P}$ allows the specification of three types of preferences. A basic desire $\varphi$ is a preference over a trajectory and therefore is a part of the basic goal language. An atomic preference is an ordering among basic desires $\Phi=\Phi_{1} \triangleleft \Phi_{2} \ldots \triangleleft \Phi_{k}$ and expresses that the preference $\Phi_{i}$ is more important than $\Phi_{i+1}$ for $1 \leq i<k-1$. An atomic preference $\Phi$ can be represented by the following goal default theory with priorities

$$
\left(\left\{\frac{T: \Phi_{i}}{\Phi_{i}} \mid i=1, \ldots, k\right\}, \emptyset, \prec_{\Phi}\right)
$$

where $\prec_{\Phi}$ is defined by $\frac{T: \Phi_{i}}{\Phi_{i}} \prec_{\Phi} \frac{T: \Phi_{j}}{\Phi_{j}}$ for $1 \leq i<j \leq k$.

A general preference is either an atomic preference or a combination of general preferences, such as $\Phi \& \Psi, \Phi \mid \Psi$, and $! \Phi$, where $\Phi$ and $\Psi$ are general preferences. Intuitively, general preferences add finitely many levels to the specification of preferences and 
thus cannot be easily represented by goal default theories which assume ceteris paribus over the preferences. Adding priorities allows only an extra layer of comparison between preferences. We view this as a weakness of goal default theories and plan to further investigate this issue.

- N-LTL and ER-LTL: These two languages allow the specification of weak and strong exceptions within goal formulae represented as LTL-formulae by introducing labels to LTL-formulae. By compiling away the labels as in [4], we can show that $\mathcal{G}_{b}$ subsumes N-LTL and ER-LTL.

Observe that the constructs used in N-LTL and ER-LTL are fairly close to default logic. This leads us to believe that interesting collections of N-LTL (ER-LTL) theories can be translated into goal default theories - which would provide a reasonable semantics for N-LTL (ER-LTL) theories with loops that have not been considered so far.

Finally, we would like to note that $\mathcal{G}_{b}$ can be easily extended to consider N-LTL (ER-LTL) formulae by

- extending $\mathcal{F}_{b}$ with N-LTL (ER-LTL) formulae; and

- extending $\mid=_{\mathcal{G}_{b}}$ to define that $\delta=_{\mathcal{G}_{b}} S$ iff $\delta=_{\mathcal{G}_{b}} c(S)$ where $c(S)$, a LTL formula, denotes the result of compiling $S$ to an LTL formula as described in $[4,5]$.

- $\pi$-CTL* and P-CTL*: These two languages consider nondeterministic domains and define goals over policies but do not consider preferences among goals. In addition, these languages introduce the operators $\mathrm{A}, \mathrm{E}, \mathrm{A}_{\pi}$, and $\mathrm{E}_{\pi}$ over paths and the two quantifiers $\mathcal{E} \mathcal{P}$ and $\mathcal{A P}$ over state formulae. Nevertheless, we can show that the $\mathrm{CTL}^{*}$ part of $\pi$-CTL* can be expressed in $\mathcal{G}_{b}$. Furthermore, $\mathcal{G}_{b}$ can be extended to allow formulae of $\pi$-CTL*. However, the two new state quantifiers are not expressible in our goal language. We observe that as the goal language is parameterized with the satisfaction relation, $\mathcal{G}_{b}$ can be easily extended with these operators. We strongly believe that these extensions will be sufficient for goal default theories with priorities to capture P-CTL*.

The above discussion highlights features from existing goal languages that can (or cannot) be expressed by our goal language. This also shows that the proposed language can serve as a unified language for evaluating goal languages. The use of default theories as the basic language also provides us with an advantage in the study of computational complexity of goal languages. In this effort, we expect that well-known complexity results on prioritized default theories [13] will be extremely useful. This will provide us with insights for the use of existing goal languages as well as the development of new goal languages.

\section{Conclusions and Future Work}

In this paper, we describe a default logic based approach to defining non-monotonic goal specification languages. We start with a basic goal specification language and use default logic (or prioritizing default logic) to provide a natural way for dealing with inconsistency and priorities over goals. We show that the new language subsumes some goal languages in the literature and can describe several features from other goal languages. We identify desirable features that cannot be easily expressed by our goal language, among them is the multi-level of preferences between goals, which we intend to investigate in the near future. We also discuss possible applications of the proposed goal language.

\section{REFERENCES}

[1] Jorge A. Baier, Fahiem Bacchus, and Sheila A. McIlraith, 'A heuristic search approach to planning with temporally extended preferences', Artif. Intell., 173(5-6), 593-618, (2009).

[2] Chitta Baral and Jicheng Zhao, 'Goal specification in presence of nondeterministic actions', in Proceedings of the 16th Eureopean Conference on Artificial Intelligence, ECAI'2004, including Prestigious Applicants of Intelligent Systems, PAIS 2004, Valencia, Spain, August 22-27, 2004, eds., Ramon López de Mántaras and Lorenza Saitta, pp. 273-277. IOS Press, (2004)

[3] Chitta Baral and Jicheng Zhao, 'Goal specification, non-determinism and quantifying over policies', in Proceedings, The Twenty-First National Conference on Artificial Intelligence and the Eighteenth Innovative Applications of Artificial Intelligence Conference, July 16-20, 2006, Boston, Massachusetts, USA. AAAI Press, (2006).

[4] Chitta Baral and Jicheng Zhao, 'Non-monotonic temporal logics for goal specification', in IJCAI 2007, Proceedings of the 20th International Joint Conference on Artificial Intelligence, Hyderabad, India, January 6-12, 2007, ed., Manuela M. Veloso, pp. 236-242, (2007).

[5] Chitta Baral and Jicheng Zhao, 'Non-monotonic temporal logics that facilitate elaboration tolerant revision of goals', in Proceedings of the Twenty-Third AAAI Conference on Artificial Intelligence, AAAI 2008, Chicago, Illinois, USA, July 13-17, 2008, eds., Dieter Fox and Carla P. Gomes, pp. 406-411. AAAI Press, (2008).

[6] M. Bienvenu, C. Fritz, and S. McIlraith, 'Planning with qualitative temporal preferences', in Proceedings of the 10th International Conference on Principles of Knowledge Representation and Reasoning (KRO6), pp. 134-144, Lake District, UK, (June 2006).

[7] G. Brewka and T. Eiter, 'Prioritizing default logic', in Intellectics and Computational Logic, volume 19 of Applied Logic Series, 27-45, Kluwer, (2000).

[8] James Delgrande and Torsten Schaub, 'Expressing preferences in default logic', Artificial Intelligence, 123, 41-87, (2000).

[9] E. A. Emerson, 'Temporal and modal logic', in Handbook of theoretical computer science: volume B, ed., J. van Leeuwen, 995-1072, MIT Press, (1990).

[10] M. Fox and D. Long, 'PDDL2.1: An Extension to PDDL for Expressing Temporal Planning Domains', Journal of Artificial Intelligence Research, 20, 61-124, (2003).

[11] A. Pnueli, 'The temporal logic of programs', in Proceedings of the 18th IEEE Symposium on Foundations of Computer Science (FOCS), pp. 46-57, (1977).

[12] R. Reiter, 'A logic for default reasoning', Artificial Intelligence, 13(1,2), 81-132, (1980).

[13] Jussi Rintanen, 'Complexity of prioritized default logics', Journal of Artificial Intelligence Research, 9, 423-461, (1998).

[14] Tran Cao Son and Enrico Pontelli, 'Planning with Preferences using Logic Programming', Theory and Practice of Logic Programming, 6, 559-607, (2006). 